\title{
Albert Soboul et la Société des Etudes Robespierristes
}

Albert Soboul and the Société des études robespierristes

Julien Louvrier

\section{(2) OpenEdition \\ 1 Journals}

\section{Édition électronique}

URL : https://journals.openedition.org/ahrf/11073

DOI : $10.4000 /$ ahrf.11073

ISSN : 1952-403X

Éditeur :

Armand Colin, Société des études robespierristes

\section{Édition imprimée}

Date de publication : 1 septembre 2008

Pagination : 209-234

ISBN : 978-2-200-92515-4

ISSN : 0003-4436

Référence électronique

Julien Louvrier, "Albert Soboul et la Société des Etudes Robespierristes », Annales historiques de la Révolution française [En ligne], 353 | juillet-septembre 2008, mis en ligne le 01 septembre 2011, consulté le 01 juillet 2021. URL : http://journals.openedition.org/ahrf/11073 ; DOl : https://doi.org/ 10.4000/ahrf. 11073

Ce document a été généré automatiquement le 1 juillet 2021.

Tous droits réservés 


\title{
Albert Soboul et la Société des Etudes Robespierristes
}

\author{
Albert Soboul and the Société des études robespierristes
}

\author{
Julien Louvrier
}

1 Faisant retour sur la carrière de l'historien des sans-culottes quelques jours après sa mort, Maurice Agulhon écrivait dans Le Monde du 14 septembre 1982 : «Albert Soboul faisait partie de ces historiens qui sont un peu des institutions tant son œuvre s'identifiait à un champ d'étude. Il avait été longtemps et il paraissait encore quelque peu l'homme de la Révolution française $»^{1}$. Le 15 septembre 1982, au cimetière du Père Lachaise, Jean-René Suratteau prononçait devant le cercueil de son ami un émouvant discours dont le premier paragraphe se concluait par ces mots : « [Soboul] était, en fait, la Société des études robespierristes, il était les Annales historiques de la Révolution française. De son pigeonnier de la rue Notre-Dame-des-Champs, il était tout à la fois $»^{2}$. La mort d'Albert Soboul scellait ainsi l'idée que l'historien avait fini par incarner la Révolution, voire personnifier les principales structures autour desquelles l'historiographie révolutionnaire s'était développée au cours du XXe siècle, et en premier lieu la Société des études robespierristes (SER).

2 Dans le contexte de sa disparition, l'évocation d'Albert Soboul comme incarnation des études sur la Révolution française et, inséparablement, de ses cadres institutionnels, n'avait rien d'incongru, elle plongeait même ses racines dans un terreau de faits vérifiables et d'éléments concrets : tout en poursuivant une carrière universitaire qui se développait nationalement et internationalement, Soboul n'avait-il pas accumulé des fonctions de direction dans la plupart des structures académiques consacrées à l'histoire de la Révolution française ${ }^{3}$ ? Sans doute est-ce à cet ensemble de responsabilités que se référaient explicitement les commentateurs à la fin de l'été 1982.

Nullement dénuée de pertinence dans les semaines qui suivirent sa mort, une telle présentation du personnage nous semble aujourd'hui difficilement acceptable tant elle renvoie à une lecture quelque peu simpliste, finaliste, voire polémique de la place qu'occupait Soboul dans l'histoire du champ scientifique que constitue l'historiographie de la Révolution française. Description rigide qui rapporte la 
trajectoire de l'historien à sa propre fin, elle est particulièrement inappropriée pour l'objet qui nous préoccupe ici. Car si l'on ne saurait réduire Soboul au réseau de structures institutionnelles et associatives qu'il a animées, on ne saurait inversement ramener ces lieux de recherche, d'enseignement ou de sociabilité, à la seule figure de Soboul. Les rapports d'Albert Soboul avec la SER se sont construits dans la pratique, hors de tout cheminement balisé. Néanmoins, les formes successives de sa relation à la communauté robespierriste ont été inévitablement façonnées par les aléas de l'histoire, du mouvement de l'historiographie et plus généralement par la nature du champ scientifique, lui-même soumis à des luttes idéologiques et politiques. Il nous revient donc de dégager la figure de l'historien d'une mise en scène figée et de lui restituer sa réalité en l'inscrivant dans la dynamique historique.

Pour autant, rendre compte de ce que furent les ambitions du chercheur, sans rien négliger des enjeux propres à la SER, n'est en aucun cas un pari gagné d'avance. D'abord parce que, s'étendant de la Libération jusqu'au début des années 1980, la période à considérer est vaste. Moment de transition, voire de recomposition de l'historiographie révolutionnaire, les quatre décennies au cours desquelles Albert Soboul a fréquenté la SER furent d'abord vécues sous le signe des certitudes : certitudes d'une histoire économique et sociale de la Révolution française comme pratique historiographique novatrice et conquérante; certitudes d'une interprétation du phénomène révolutionnaire déterminée par l'idée de l'avènement de la bourgeoisie et du capitalisme; certitudes idéologiques du militant communiste quant à la valeur scientifique du marxisme ; certitudes enfin quant aux grands principes déontologiques et méthodologiques à la base du métier de l'historien imposant à tous les prétendants à la carrière le travail des sources et la rédaction d'une thèse. Mais ces années furent également celles des grands débats historico-idéologiques des années de guerre froide autour du " mythe » cobbanien ${ }^{4}$ ou de la "Révolution atlantique ${ }^{5}-$, et des remises en questions plus profondes - que l'on pense à l'apparition de la notion de "dérapage ${ }^{6}$ choisie par François Furet et Denis Richet pour stigmatiser le cours de la Révolution à partir de l'année 1792 et à celle de "catéchisme révolutionnaire $»^{7}$ élaborée par François Furet seul pour dénoncer l'historiographie d'inspiration marxiste. À la difficulté posée par l'appréhension d'une période longue et riche en rebondissements, vient s'ajouter l'absence de sources caractérisées ${ }^{8}$ et la faiblesse relative des enquêtes historiques consacrées aux historiens de la Révolution française en particulier et aux sociétés d'histoire à l'époque contemporaine en général ${ }^{9}$. Dans le cas de la SER, cette pauvreté relative s'explique en grande partie par le fait que malgré sa stabilité, son activité pérenne ${ }^{10}$ et les moyens dont elle disposait du temps où elle se trouvait -en dehors de tout cadre officiel - adossée à l'Institut d'histoire de la Révolution française (IHRF), elle n'a pas su se constituer de fonds d'archives. Quant aux enquêtes sur Albert Soboul, elles ont porté sur son travail de thèse, sur ses positions historiographiques, mais plus rarement sur sa trajectoire de jeune chercheur, ses années de formation et d'apprentissage, sur les effets socialisateurs de son engagement communiste précoce et les répercussions de cet engagement sur son début de carrière. En fait, faute de sources bien identifiées ${ }^{11}$ et peut-être aussi de la distance nécessaire - vingt-cinq ans seulement se sont écoulés depuis sa mort - les historiens qui se sont intéressés à la vie d'Albert Soboul se sont concentrés sur quelques épisodes célèbres et sur certains travaux remarquables et n'ont pas pu ou pas voulu mener plus loin leurs investigations biographiques $^{12}$. 
5 L'état actuellement limité de la recherche a pour conséquences, d'une part, de laisser dans l'ombre les jeunes années de la vie de l'historien jusqu'à sa soutenance de thèse en 1958, d'autre part, de laisser se perpétuer les images qu'ont construites de lui ses amis et ses adversaires dans un contexte de polémique, de combat politique ou de commémoration, et qui trop souvent ont rapporté son parcours et ses choix historiographiques à des " postures ", des entités collectives ou à des catégories figées.

6 L'étude des rapports entre Albert Soboul et la SER doit se défaire de ces éclairages "polémiques ", «militants» ou "commémoratifs", qui, plutôt que de révéler la complexité du personnage, en occultent les nuances et les ombres comme le font parfois les projecteurs de théâtre quand ils sont mal réglés. Cela implique de faire retour aux origines de son engagement d'historien dans la SER et de retrouver ce que furent ses propres ambitions et ses propres objectifs tels qu'ils se sont construits et modifiés au cours de toute une vie d'enseignement et de recherche. Toutefois, afin d'éviter de tomber à notre tour dans les mêmes travers que ceux que nous dénonçons, notre démarche visera d'abord à analyser de façon critique les différentes constructions discursives dont la figure de Soboul a fait l'objet. Autrement dit, nous nous proposons d'essayer de circonscrire ce dont Soboul est le nom; ie ce à quoi renvoie le nom «Soboul » dans l'imaginaire des historiens de la Révolution française, de la communauté des historiens et du public français et étranger à l'horizon des années 1980. Il nous sera ainsi possible d'évoquer de façon plus objective ce que furent réellement sa place et son action au sein de la Société des études robespierristes.

\section{Ce dont Soboul est le nom : un regard critique}

7 Tout discours qui se donne pour objet d'apprécier ce qu'était ou ce que fut l'insertion d'Albert Soboul dans la vie scientifique et intellectuelle devrait s'appuyer prioritairement sur des critères en rapport avec son activité de recherche et son métier d'enseignant. On mesurerait ainsi l'apport de l'historien d'abord à l'aune de ses ouvrages et de ses nombreux articles, glanes, notices, chroniques, avant-propos et comptes rendus publiés tout au long de sa vie dans les Annales historiques de la Révolution française, Annales ESC, La Pensée, L'Information historique, parfois repris à l'étranger, par exemple dans Past \& Present en Grande-Bretagne ou Passato epresente en Italie. En soi, l'évaluation quantitative et qualitative de la production scientifique d'Albert Soboul ne pose pas de problème ${ }^{13}$. Pourtant, il n'en est que rarement question dans les textes qui lui ont été consacrés, sinon accessoirement ou partiellement. En fait, on peut facilement montrer que la plupart des discours sur Soboul comportent une large part d'arguments d'autorité et de qualificatifs connotés, bref qu'ils s'appuient davantage sur un légendaire, tantôt favorable tantôt défavorable, que sur une prise en charge globale et érudite de l'homme et de son travail. Pour éviter pareil écueil, commençons par rappeler quels sont les principaux stéréotypes qui ont été accolés au nom de Soboul dans le discours de ses contempteurs d'abord, dans celui de ses proches ensuite ${ }^{14}$.

\section{Albert Soboul en gardien d'une orthodoxie}

8 Le premier type de discours qui nous intéresse, appelons-le " discours de polémique ", se caractérise par la stigmatisation de toutes les facettes saillantes du personnage, un peu comme le font les caricaturistes : ainsi, en prenant prétexte de la foi solide d'Albert 
Soboul dans l'institution universitaire républicaine, on a pu donner de lui l'image d'un " mandarin "; en évoquant son engagement communiste jamais renié on a parlé de lui comme d'un «stalinien»; et enfin, dans son attachement au marxisme on a vu le signe $\mathrm{du}$ « dogmatisme ». Mais par-dessus tout, ce discours a cherché à enfermer Soboul tout entier dans la figure du gardien intransigeant d'une orthodoxie historiographique incompatible avec le progrès de la connaissance scientifique. Revenons sur les trois temps qui ont rythmé son élaboration.

9 Le « discours de polémique » est apparu initialement en 1971 sous la plume de François Furet dans un article programmatique intitulé « Le catéchisme révolutionnaire »" Cet article fut rédigé après que le livre La Révolution française, célèbre histoire illustrée cosignée par Furet et Denis Richet, eut été sérieusement critiqué par Claude Mazauric et Albert Soboul pour ses jugements hâtifs et son ton prétentieux ${ }^{16}$. Les expressions choisies alors par Furet pour disqualifier les travaux sobouliens d'histoire révolutionnaire sont bien connues. Commentant l'état de l'historiographie, il écrivait : "Ainsi s'est constituée, au niveau de l'interprétation de la Révolution française, cette sorte de vulgate lénino-populiste dont Le Précis de Soboul est sans doute le meilleur exemple $\aleph^{17}$. Cet ouvrage, poursuivait-il, est l'illustration d'une historiographie à « l'esprit manichéen, sectaire et conservateur $»^{18}$, « qui substitue le jugement de valeur au concept, la finalité à la causalité, l'argument d'autorité à la discussion " ${ }^{19}$. Quelques lignes plus loin, Soboul se voit attribuer une conscience historique «à la fois commémorative et finaliste $»^{20}$, avant d'être qualifié de «théologien $»^{21}$ et accusé finalement de n'avoir à proposer de l'histoire de la lutte des classes qu'une vision simpliste $^{22}$.

On peut considérer qu'à partir de 1971, le vocabulaire, les différents registres de dénigrement, en somme les grandes lignes du réquisitoire de Furet contre Soboul sont fixées. Désormais il n'est plus nécessaire de discuter des travaux historiques dans leur spécificité, pour peu qu'ils semblent s'inscrire dans la tradition républicaine favorable à la Révolution et qu'ils utilisent des concepts issus de la pensée marxiste, il est possible d'évoquer directement une "vulgate ", ou plutôt un "catéchisme ", auxquels on a attaché un nom. Pour faire de cette rhétorique une évidence incontestable, rien de plus simple que de marteler son message. François Furet s'y emploie dès 1978 en faisant reparaître son article, complété de quelques autres, dans le recueil Penser la Révolution française ${ }^{23}$.

11 Avec la mort de Soboul en 1982, le "discours de polémique " franchit une deuxième étape. En reprenant à leur compte les arguments à l'instant évoqués, de grands médias nationaux installent un peu plus dans l'opinion l'image de Soboul élaborée par François Furet de l'homme rigide, guidé par une croyance aveugle en un horizon idéologique qui interdit toute intelligence du phénomène révolutionnaire. L'exemple le plus frappant en est donné par l'article nécrologique que publie sans signature le Nouvel observateur ${ }^{24}$. Pour retracer la trajectoire d'Albert Soboul et esquisser son apport à l'historiographie révolutionnaire, l'auteur - il s'agit du philosophe Jean-Paul Enthoven - ne s'embarrasse pas de la longue bibliographie de l'historien. Les recherches de Soboul sont tout simplement réduites à sa thèse et sa carrière rapportée à une série de doctorats honoris causa des grandes universités des pays d'Europe de l'Est. Le chroniqueur est davantage préoccupé par le communisme du spécialiste de la Révolution que par son travail de chercheur : «Soboul n'était pas seulement un historien. Il était aussi, depuis les années trente, membre d'un PCF dont la vigilance en matière d'historiographie révolutionnaire 
ne s'est jamais démentie ». Il importe peu au journaliste de prendre la mesure exacte de ce que fut l'investissement de Soboul dans le Parti communiste ou d'accorder la moindre importance aux signes visibles de son indépendance d'esprit ${ }^{25}$. Après avoir dans un premier temps estampillé Soboul du sceau de la vulgate, le "discours de polémique » entend dorénavant attacher à son nom et à ses travaux historiques, la marque infamante de la fidélité à un parti. À cet égard, soulignons que le « discours de polémique » prend place dans une vaste entreprise de déconsidération du PCF initiée dès le début des années 1970 au nom du combat antitotalitaire par un grand nombre d'intellectuels issus de la gauche non-communiste ${ }^{26}$ et renforcée par les tensions entre le PS et le PCF après la rupture du programme commun et les mauvais résultats de la gauche aux élections législatives de 1978. Par conséquent, on ne peut saisir le « discours de polémique » à l'endroit de Soboul que dans le contexte plus large d'une pratique systématique de disqualification ${ }^{27} \mathrm{du}$ communisme à grande échelle qui touche non seulement au PCF, mais plus largement à tout ce qui légitime son existence dans la société française, que ce soit la catégorie de "classe ouvrière ", celle de "mouvement ouvrier ", ou encore ses plus célèbres figures, dirigeants politiques, intellectuels de parti et compagnons de route ${ }^{28}$.

Dans sa formulation ultime, le « discours de polémique » a intégré l'idée de l'expulsion complète d'Albert Soboul de l'histoire de l'historiographie révolutionnaire. Le chapitre historiographique du Dictionnaire critique de la Révolution française paru à l'occasion du bicentenaire atteste ce mouvement de façon saisissante ${ }^{29}$. François Furet y évoque Albert Mathiez dont il stigmatise les origines modestes, tient compte de Georges Lefebvre qu'il présente comme "un positiviste de la vieille école » que la Libération aurait transformé en "néophyte du marxisme-léniniste», puis le texte s'arrête. En mettant un point final au développement de « l'histoire universitaire de la Révolution française " après la mort de Georges Lefebvre, François Furet ne fait rien d'autre qu'évincer Soboul de l'historiographie. Loin d'adoucir le "discours de polémique », ce procédé n'en rend la portée que plus grande et renforce l'idée de la personnification de toute une partie de l'historiographie révolutionnaire dans la figure de l'historien des sans-culottes ${ }^{30}$.

13 Faut-il s'étendre sur l'intérêt qu'il pouvait y avoir, dans le contexte de la préparation de la commémoration du bicentenaire de 1789, à réduire l'histoire qu'on enseignait à la Sorbonne et qui trouvait sa place dans les pages des AHRF, aux positions d'un seul homme? Pareille présentation devait rendre légitime et crédible l'opération de relecture de l'histoire de la Révolution française initiée par François Furet ${ }^{31}$.

\section{Albert Soboul en champion d'une tradition}

En contrepoids au "discours de polémique » qui semble épouser étroitement la chronologie des débats idéologiques au sein de la gauche française dans la deuxième moitié du $\mathrm{XX}^{\mathrm{e}}$ siècle, se déploie une autre façon de parler d'Albert Soboul, favorablement cette fois. Élaborée par ses disciples, ses amis ou ses proches, elle constitue l'espace d'expression de deux grands types de discours dont les enjeux dépassent largement la stricte évaluation de l'œuvre soboulienne: "discours de combat» d'une part, dont l'objet est de riposter au "discours de polémique ", « discours de commémoration » d'autre part, à usage principalement interne destiné à entretenir la mémoire de la communauté robespierriste. Bien que souvent plus nuancées que le " discours de polémique », ces lectures bienveillantes n'aident pas pour 
autant à distinguer clairement qui fut vraiment Albert Soboul. En effet, qu'il s'agisse de célébrer sa mémoire ou de la défendre, elles tendent toutes deux à rapporter le personnage à la tradition historiographique dont il revendiquait l'héritage. Ce faisant, elles négligent de faire entendre ses doutes et ses interrogations, tout en lissant les aspérités de son caractère.

Deux types de documents permettent de mieux cerner ces discours : d'une part, le livre de Claude Mazauric publié en 2003, Albert Soboul, Un historien en son temps. Essai de biographie intellectuelle et morale ${ }^{32}$, que nous avons rangé dans la catégorie des « discours de combat »; et d'autre part, l'hommage prononcé par Jean-René Suratteau en tant que représentant de la SER aux obsèques d'Albert Soboul en septembre 1982 qui relève, lui, $\mathrm{du}$ " discours de commémoration ». Chacun à leur manière, ils font émerger une figure singulière d'Albert Soboul : celle du «Soboul œcuménique » chez Mazauric, celle du «Soboul institutionnalisé » chez Suratteau ${ }^{33}$.

\section{La figure du Soboul œcuménique par Claude Mazauric}

16 Enquête roborative fondée sur un certain nombre de documents inédits, de correspondances et de souvenirs personnels, l'essai biographique de Claude Mazauric est doté de tous les attributs de l'étude érudite et scientifique. Toutefois, derrière le ton amical et chaleureux se dessinent les contours d'un projet militant de réhabilitation d'Albert Soboul, de son œuvre et à travers elle, de défense des acquis de l'historiographie "scientifique " ${ }^{34}$ de la Révolution française.

17 Arracher le nom de Soboul à l'image dans laquelle le «discours de polémique » l'a enfermé - c'est tout l'enjeu du « discours de combat » -, implique de répondre point par point aux attaques dont l'historien a fait l'objet, qu'il s'agisse de la mise en cause de son marxisme, de ses engagements politiques, de la dénonciation de son dogmatisme historiographique voire de son autoritarisme. Mais en s'attachant prioritairement à l'examen de ces objets, en reprenant de fait la grille de lecture imposée par le "discours de polémique ", Claude Mazauric se prive inévitablement de la possibilité d'aborder d'autres problématiques non moins capitales pour saisir la trajectoire de l'historien. Ainsi, le rôle réellement joué par Albert Soboul dans les polémiques historiographiques, ses difficultés à s'ouvrir aux nouvelles voies de la recherche - sa méfiance, par exemple, à l'égard des travaux de Michel Vovelle - ou encore son attitude face aux débats qui marquèrent son propre camp politique ${ }^{35}$, ne sont que très brièvement abordés. On cherche ici à favoriser des aspects plus consensuels de la vie de Soboul, qu'illustrent les nombreuses listes de personnalités ${ }^{36}$ qui ponctuent l'ouvrage, comme autant de preuves de l'ouverture d'esprit et de la tolérance de «l'homme Soboul $\aleph^{37}$. Mobilisée pour la démonstration, pareille collection de noms propres vise à associer au nom Soboul l'idée d'un certain œcuménisme propre à donner de lui l'image d'un homme de dialogue, occupant le centre d'un réseau international de recherche aux tendances parfois très différentes ; bref, un Soboul quasi irénique, diamétralement à l'opposé de la figure de chef de fil rigide d'une école réduite à une vulgate et repliée sur elle-même, telle que la renvoyait le " discours de polémique ».

Dans le même esprit, la construction du Soboul œcuménique s'appuie sur une lecture compréhensive de son caractère. Claude Mazauric tempère les colères de cet homme sanguin qui avait le souci de tout diriger, en dressant de lui le portrait d'» un organisateur absolument dévoué à sa tâche » dont il faut mettre les «manières de 
despote éclairé qui contrôlait toutes les manettes » sur le compte de la conjoncture : des façons de faire nécessaires sans lesquelles « dans le contexte de l'époque tout aurait sombré dans le chaos ${ }^{38}$. La biographie de Claude Mazauric tend donc à présenter un Soboul au caractère plus lisse, plus facile, plus accessible. Cette image complète et nuance idéalement la figure du Soboul institutionnalisé diffusée par la SER le jour de ses obsèques.

\title{
La figure du Soboul institutionnalisé par Jean-René Suratteau
}

Une des spécificités du "discours de commémoration » consiste à faire porter sur les épaules de Soboul le poids de toute une histoire, voire de toute une tradition. Dans ce cas précis, il se caractérise par une rhétorique réifiante qui rapporte l'historien aux structures qu'il a dirigées, à l'exemple du discours prononcé par Jean-René Suratteau devant le cercueil de Soboul en septembre 1982 :

\begin{abstract}
«Albert Soboul n'était pas seulement membre de la Présidence de la Société des études robespierristes depuis la mort en 1959 de Georges Lefebvre, il n'était pas seulement le Secrétaire général de cette société, il n'était pas seulement le Directeur, le rédacteur en chef des Annales historiques de la Révolution française - dont il était aussi le correcteur -, il était, en fait, la Société des études robespierristes, il était les Annales historiques de la Révolution française. De son pigeonnier de la rue Notre-Dame-des-Champs, il était tout à la fois. Il était de ceux qui s'étaient opposés il y a une quinzaine d'années au changement de nom de la Société, fidèle ainsi à Albert Mathiez et à Georges Lefebvre $»^{39}$.
\end{abstract}

Dans ce texte, la redondance des fonctions, des intitulés, des positions occupées par Soboul est au service de l'idée qu'il personnifiait concrètement, c'est-à-dire pas seulement par ses livres, mais également par son activité quotidienne, donc de façon dynamique, l'historiographie de la Révolution ${ }^{40}$. L'hommage de Suratteau va jusqu'à faire de Soboul la figure idéale de l'historien de la Révolution française : « Il incarnait, nonobstant les critiques et les attaques de quelques-uns, dont la trajectoire politique accentuée s'irritait de sa fidélité, l'historien de la Révolution française aux yeux de l'énorme majorité de ceux qui s'intéressaient à cette histoire [...] ». Tel qu'il se présente ici, le "discours de commémoration" pose deux séries de problèmes à l'historien de l'historiographie. D'abord, il comporte en lui une tendance à réduire la SER et avec elle l'historiographie érudite, à un seul homme. Cela revient à occulter les différents courants interprétatifs qui coexistaient dans la Société et dans l'historiographie du vivant de Soboul. Une telle présentation participe involontairement de la mythologie construite par Furet à travers le "discours de polémique». Ensuite, le "discours de commémoration", au même titre que le "discours de combat", contribue à faire de Soboul le produit d'une tradition historiographique dont on peut facilement démontrer qu'il a largement contribué luimême à en forger la réalité discursive au cœur des polémiques des années 1960 et 1970, donc à un moment où le combat idéologique rendait nécessaire de rassembler autour des grandes figures "de Michelet à Lefebvre $»^{41}$, des orientations parfois très disparates. On peut se demander s'il ne serait pas plus pertinent de relativiser l'inscription de Soboul dans cette logique commémorative - en la rapportant notamment au fonctionnement des luttes politiques dans lesquelles elle s'inscrivait pour faire émerger un discours de la complexité, porteur d'une lecture plus autonome de la conception de la Révolution française qui était la sienne. 
Ces remarques posées, essayons maintenant de décrire quelle fut la réalité de l'investissement d'Albert Soboul dans la vie de la SER.

\section{Dans l'ombre de Lefebvre : I'heure des accomplissements (1945-1959)}

Au début des années 1950, une nouvelle génération d'historiens de la Révolution est en train d'émerger. Sous la figure tutélaire de Lefebvre et avec l'intention d'étudier le peuple des villes dans la perspective de "l'histoire vue d'en-bas», Albert Soboul se retrouve aux côtés des Anglais Richard Cobb et George Rudé, ou encore du norvégien Kâre Tonneson. À la différence de la plupart des membres qui composent alors la SER, cette génération n'a pas connu Mathiez. Au sein de cette jeune équipe, comme au sein de la SER, Soboul, professeur agrégé d'histoire, militant communiste, est un chercheur en quête de légitimité.

\section{Des prises de responsabilité progressives : les années de la Libération}

Le nom d'Albert Soboul apparait pour la première fois dans les AHRF en 1946. Il figure sur la liste des membres de la SER ayant répondu à la circulaire de 1945 envoyée dès la fin de la guerre pour régulariser le fichier des adhérents. Toutefois, la véritable introduction de Soboul dans les milieux robespierristes est à chercher dans les comptes rendus qui paraissent dans les AHRF cette année-là : signée par Georges Lefebvre, une note des plus flatteuses signale en effet l'intérêt du recueil 1789. L'An I de la libertét ${ }^{42}$ préparé par Soboul à l'occasion du cent-cinquantenaire de la Révolution : "Je crois que c'est son premier ouvrage, je le regarde avec plein de promesses $»^{43}$.

Sous l'impulsion de Lefebvre, la reprise d'activité de la Société a été rapide, ses membres ont pu recommencer à se réunir régulièrement. Les procès-verbaux des assemblées générales indiquent que Soboul s'y montre assidu. En juin 1946, il manifeste le désir de participer activement aux chantiers de la SER en se portant volontaire pour établir la nouvelle table analytique des AHRF en collaboration avec Henri Calvet. L'année suivante, Lefebvre indique vouloir relancer le travail initié par Mathiez dans la perspective de la publication des Cuvres complètes de Robespierre ${ }^{44}$. Soboul, quoiqu'encore largement engagé dans des activités militantes accepte de prendre à sa charge une partie du travail ${ }^{45}$. L'année suivante, il donne aux AHRF son premier $\operatorname{article}^{46}$.

Il faut savoir qu'à la fin des années 1940, l'activité de recherche de Soboul est encore peu distincte de ses engagements politiques. Elle est ponctuée de nombreuses interventions à l'extérieur du cercle des spécialistes de la Révolution française, et particulièrement de travaux de vulgarisation pour les journaux et publications liés au Parti communiste ${ }^{47}$. Ces écrits sont en grande partie des œuvres de commande qui parfois ne concernent pas directement l'histoire révolutionnaire, sinon de loin, comme cette brochure consacrée à la Révolution de 1848 qui paraît en $1948^{48}$. C'est tout de même à l'histoire de la Révolution française que Soboul consacre son premier manuel, une synthèse composée à la demande des Éditions sociales ${ }^{49}$. Simplement intitulé $L a$ Révolution française, ce livre, qui constituera la base du futur Précis, est rapidement 
traduit et lui ouvre les portes de l'Europe. En avril 1950, Soboul voyage en Tchécoslovaquie pour une série de conférences qui l'emmène à Brno et à Prague ; en janvier 1951 il parle quatre soirs de suite à Genève et en janvier 1952 il est en Italie. Il n'est pas exagéré de dire qu'une part non négligeable de sa notoriété internationale fut acquise dès le début des années 1950, bien avant la publication de ses thèses et de la plupart de ses travaux strictement académiques. Ceci étant dit, les premiers articles qui paraissent dans les $A H R F$ et le soutien renouvelé de Georges Lefebvre apportent à Soboul la légitimité scientifique et la reconnaissance de la communauté robespierriste $^{50}$. Juste avant l'été 1949 il est logiquement élu au Comité directeur de la SER dont il devient le secrétaire-adjoint ${ }^{51}$.

\section{L'homme d'une nouvelle génération}

27 L'analyse des débats qui traversent la Société au cours de la période suivant l'arrivée de Soboul dans les instances de direction jette une lumière crue sur une époque où une réelle distance sépare déjà deux générations de membres d'une part et deux conceptions du rôle de la SER d'autre part. En effet, à l'exemple de Soboul, les nouveaux venus n'ont pas vécu, sinon de loin, la lutte sans merci qui a vu s'opposer Aulard et Mathiez. Leur attachement à la figure du père fondateur et aux combats qu'il a incarnés compte moins que leur souci de trouver un cadre où exposer les résultats de leurs recherches. Par ailleurs, au fur et à mesure que s'éloigne l'enjeu posé par Mathiez de la " connaissance de Robespierre », la SER attire à elle des spécialistes de la Révolution qui entendent qu'elle ne se préoccupe que d'histoire de la Révolution et qu'elle se tienne dorénavant éloignée des questions du présent, comme celle de la réhabilitation de Robespierre.

Soboul, lui, semble respecter une règle stricte faite d'indépendance scientifique quand il rédige ses travaux et de fidélité à la tradition quand il s'agit de la vie de la SER. S'il s'engage dans la préparation du recueil d'articles de Mathiez, Études sur Robespierre, que publie la SER à l'occasion du bicentenaire de la naissance de l'Incorruptible, il n'en conserve pas moins une véritable indépendance historiographique dont témoigne d'ailleurs un échange de courrier publié dans les AHRF en 1958. Accusé par Jean Dautry, ancien élève de Mathiez, de manquer de déférence à l'égard des travaux mathieziens sur la déchristianisation, Soboul défend son droit à s'en émanciper au nom de l'approfondissement des connaissances historiques ${ }^{52}$. Les divergences entre les membres de la SER ne concernent pas tant les interprétations de la Révolution que la définition de l'objet même de la Société. À la mort de Lefebvre, quand se pose le problème de sa succession, surgissent de réelles craintes. Les correspondances échangées tout au long de l'année 1959, par Robert Schnerb et Maurice Dommanget ou par Albert Soboul et Jean-René Suratteau, reviennent systématiquement sur l'enjeu que constitue la préservation de l'identité «mathiezienne » et donc « robespierriste » de l'association.

Dès mars 1959, alors que Lefebvre, fatigué, a annoncé son intention de se retirer de toutes ses fonctions et responsabilités, Robert Schnerb fait part de ses inquiétudes à Maurice Dommanget : «Bien que je n'aie rien personnellement à reprocher à Reinhard, que je tiens pour un collègue amène, je suis bien décidé à m'en aller si l'œuvre de Mathiez tombe sous la main d'un clérical quelconque. Pauvre société ! Elle risque bien de se disloquer! Pauvre Robespierre, qui n'a décidément pas de chance ! ${ }^{53}$ À la mort de 
Lefebvre, dont l'autorité incontestable garantissait l'intégrité de la SER, la question identitaire se pose de façon plus aiguë. Voici ce qu'écrit René Garmy à Dommanget en septembre 1959: «La mort de G.L. est une perte immense pour l'histoire de la Révolution française et pose, comme tu l'indiques, de graves problèmes pour l'avenir de la Société [...]. Je ne vois guère d'autre personnalité que Labrousse pour maintenir l'unité et l'esprit de la Société. Soboul est également de cet avis $»^{54}$. Dans une lettre à Suratteau datée du 8 septembre 1959, Soboul précise sa position en rappelant d'abord son attachement à son maître : « La mort du Vieux est pour nous, les gens comme toi et moi, un coup très dur à plus d'un titre. Quelles que soient les réserves que l'on puisse faire sur l'homme, son caractère, ses relations avec ses amis, c'était un grand bonhomme irremplaçable. Il laisse dans l'histoire de la Révolution, un vide immense ${ }^{55}$. Abordant finalement la question de la succession à proprement parler, Soboul, qui affirme ne voir " personne en ce moment pour remplacer [Lefebvre] dignement ", fait la liste des prétendants : «Reinhard, qui, il y a trois ou quatre ans, n'était même pas membre de la société ? [...] Godechot l'atlantique ? [...] Bouloiseau se démène, paraît-il, pour une solution de compromis (qui sauvegarderait son fromage personnel) [...] quant à moi, je pense que Labrousse est le seul digne ».

Soboul a côtoyé Labrousse en maintes occasions et connaît ses engagements politiques et son attachement à la Révolution. Il sait aussi combien Lefebvre en était admiratif. En optant pour une direction collégiale composée de quatre co-présidents: Labrousse, Reinhard, Godechot, Soboul, les membres du Comité directeur de la Société choisissent de préserver les liens de celle-ci avec l'IHRF dont Marcel Reinhard vient d'être nommé directeur, sans la laisser toutefois aux seules mains du titulaire de la chaire de la Sorbonne. Loin de l'image d'une paisible société d'admiration mutuelle, la fin de la " période Lefebvre » démontre à quel point, dès cette époque, la SER est un lieu où se jouent des luttes de pouvoir qui sont aussi des luttes symboliques pour le monopole de la légitimité historiographique. Après avoir patiemment gravi les échelons d'une ascension dont il n'a brûlé aucune étape, commence pour Soboul une période d'intense activité et d'investissements d'un genre nouveau. À l'ère des accomplissements, celui de la thèse, des articles, de la conquête silencieuse de la reconnaissance de ses pairs, succède la période de l'engagement et de l'exposition. Co-président et secrétaire général de la SER en charge des AHRF, il doit affronter une scène historiographique en voie de recomposition. Pour Soboul, le temps des responsabilités et des combats est aussi celui des désillusions.

\section{Le temps des responsabilités et des désillusions (1959-1982)}

Une fois élu à présidence de la SER, l'activité scientifique de Soboul, toujours plus importante, se double de lourdes responsabilités que renforce encore son élection en Sorbonne en $1967^{56}$. La décennie 1958-1968 voit sa production de nature spécifiquement militante - ici partisane -, encore relativement importante à la fin des années 1940 et au début des années 1950, s'épuiser peu à peu, sans qu'il soit toutefois possible de dater précisément le moment d'un désinvestissement net. Il n'est pas exagéré de faire remonter le déclin de l'activité militante de Soboul, en faveur d'un réinvestissement dans le travail académique, à la reconversion vers l'enseignement secondaire opérée très rapidement après son échec à occuper un poste dans la haute administration à la 
Libération, la transition vers une vie dédiée entièrement à la production académique s'accentuant au cours des années 1950 à mesure que la fin de rédaction de sa thèse approchait. Dans la dernière période de sa vie, et particulièrement vers le milieu des années 1970, Soboul est confronté aux désillusions et à la lassitude. La SER évolue peu, ses membres sont peu enclins à ouvrir le débat pour repenser collectivement le manifeste qui gouverna à sa création et faute de visée claire, elle ne cesse d'osciller entre expertise et engagement, entre société savante et société de pensée. Dans un contexte où la pression de la polémique rend l'atmosphère pesante ${ }^{57}$, épuisé par les attaques incessantes dont il fait l'objet et qui minent ses dernières années ${ }^{58}$, Soboul impose à la Société comme à la revue, tout à la fois la responsabilité de l'héritage historiographique, c'est-à-dire la tradition du combat robespierriste, la fidélité à l'œuvre de Mathiez et Lefebvre, et une ouverture progressive et limitée aux nouveaux chantiers. Avant d'examiner l'effet des controverses sur le rapport de Soboul à la SER, revenons pour commencer sur la façon dont il concevait la place de la SER dans le champ intellectuel et son propre rôle d'animateur des AHRF.

\section{Comment Albert Soboul concevait-il son rôle de secrétaire général de la SER ?}

Tout juste élu à la présidence collégiale de la SER, Albert Soboul s'empresse d'exposer ses ambitions pour la revue dans un courrier à Suratteau. Plus que de simples pistes de travail, on peut parler d'un véritable programme pour faire entrer la publication dans une ère nouvelle, en s'attachant particulièrement à tenir compte de l'internationalisation croissante de la recherche sur la Révolution française :

"Je conçois la rédaction des Annales comme un travail d'équipe. Donc je mobilise

les amis : Cobb, Dautry, toi... Il faut que tu envoies articles, glanes, notices, compterendus, revues de presse... tout ce qui peut rendre une revue vivante. $\mathrm{Tu}$ lis l'allemand "comme père et mère" : je te ferai parvenir tous les livres teutons. Et tu peux faire tous les compte-rendus que tu voudras [...]. Il faut des compte-rendus, beaucoup de compte-rendus $»^{59}$.

33 L'état d'esprit volontariste du nouveau directeur de la revue s'accompagne d'un haut niveau d'exigence vis-à-vis de ses collaborateurs. La correspondance de Soboul avec Suratteau révèle qu'il ne cesse, jusqu'à la fin de sa vie, d'être particulièrement exigeant avec les auteurs, encourageant les uns, pressant les autres, relisant et corrigeant chaque article, courant après les comptes rendus et les chroniques. Outre son implication dans l'animation et la conception des $A H R F$, dont il augmente la pagination et diversifie les contributions, Soboul entend aussi influer sur le devenir de la SER ${ }^{60}$. Il soutient, non sans difficulté, le programme défini par Mathiez. Cela se traduit par la poursuite de la publication des Euvres complètes de Robespierre, et surtout par un attachement à l'identité expressément « robespierriste » de l'association dont il refuse catégoriquement le changement de nom. Enfin, sans rien céder sur les principes qui présidèrent à sa création, Soboul souhaite faire de la SER un lieu d'échanges qui ne se limiterait pas aux universitaires.

Il ne faut pas réduire la question du changement de nom à une affaire formelle. Régulièrement débattue, elle fut reposée de façon originale par Marcel Reinhard au cours de l'année 1970. Profitant de l'occasion qui lui était fournie par la rédaction du compte rendu d'une brochure de René Sidersky, un ancien élève d'Aulard installé en Argentine et membre de la SER depuis de nombreuses années, Reinhard écrivait : «M. 
Sidersky [...] propose d'élargir le nom de la Société des études robespierristes, pour en faire une "Société d'histoire de la Révolution française". Cette invitation mérite d'être retenue, elle a été faite déjà par divers historiens et à plusieurs reprises, elle correspond plus exactement aux activités de la société " ${ }^{61}$. Soboul et Godechot s'opposèrent fortement à ce projet que défendaient principalement l'entourage de Reinhard et des membres étrangers de la SER. Un vote consultatif fut organisé au cours de l'assemblée générale du 20 juin 1971 et l'idée fut rejetée.Au cours des années 1970, Soboul et son entourage ont donc refusé de faire de la SER une compagnie de techniciens ou d'experts de la Révolution française, restant en cela fidèles aux orientations de Mathiez. Il faut souligner que l'adhésion de Soboul à la formule imaginée par le fondateur de la Société n'avait rien de circonstancielle. Déjà, à la fin de l'année 1959, il confiait à Suratteau : "Notre société est aussi une société : on l'oublie, unie par l'attachement à Robespierre. Quelle que soit l'inégale valeur scientifique des uns ou des autres, nous leur devons une égale amitié, si nous les estimons fidèles au but de la Société ${ }^{62}$. Son rapport de secrétaire général du 11 mars 1962 insistant sur «la nécessité d'orienter le recrutement de la Société, conformément aux vues de son fondateur, en particulier vers les milieux non-universitaires $»^{63}$, confirme la constance de ses orientations.

Dans le même esprit, Soboul s'est engagé dès son élection à garantir à la SER une activité régulière. Il s'agit principalement de veiller à ce que chaque assemblée générale de la Société soit l'occasion d'entendre un chercheur présenter ses travaux. Dans une lettre envoyée à Suratteau au début de l'année 1961, Soboul livre un témoignage capital sur les résistances qui s'élèvent face à sa détermination à préserver la SER comme un lieu de sociabilité quand d'autres voudraient qu'elle se concentre sur sa seule activité éditoriale : «[...] Les pontifes se fichent de la Société comme de leur première chemise. Et Bouloiseau se charge de freiner tout, pour ne pas dire saboter, avec la franchise qui le caractérise. Comme eux ne font rien, ils ne veulent pas que je fasse quelque chose et que je paraisse donner vie et activité à la Société ${ }^{64}$.

Malgré de vraies difficultés, ni les AHRF ni les rencontres de la Société ne semblent avoir souffert d'aucune baisse de régime au cours de la période 1959-1982. Ce dynamisme est à mettre sur le compte du talent et de la persévérance de Soboul. Outre son réseau international fourni, qui s'étend encore à partir de sa nomination en Sorbonne et qu'il entretient par de très nombreuses missions à l'étranger, Soboul dispose dès 1971 avec son séminaire de recherche, d'un formidable réservoir d'idées et de talents. Pour alimenter la revue et animer les assemblées générales de la SER dont il est le grand organisateur, Soboul puise dans les travaux de ces jeunes chercheurs qui travaillent autour de lui à l'IHRF et dont il dirige les thèses. Même s'il n'en est pas à l'initiative, Soboul accompagne les évolutions alors en cours dans l'historiographie révolutionnaire, il leur donne, parfois avec retard, une visibilité certaine.

\section{Entre gestion de l'héritage historiographique et nouvelles perspectives : la ligne éditoriale des Annales historiques de la Révolution française}

37 La ligne éditoriale des AHRF au cours de la période Soboul (1959-1982), pour autant qu'elle se laisserait apprécier en quelques lignes, témoigne d'une double volonté : respecter et défendre l'héritage historiographique dont lui-même se reconnaît le 
légataire, à savoir l'œuvre de Mathiez et Lefebvre, et au-delà celle de Jaurès ; ouvrir de façon mesurée les pages de la revue aux voies nouvelles de la recherche. Deux dixièmes de la production d'Albert Soboul dans les AHRF sont consacrés à célébrer la mémoire des grandes figures de l'historiographie révolutionnaire. De cette mémoire, Soboul a fixé définitivement les termes à l'occasion d'un hommage à Georges Lefebvre :

«Dernière des grandes histoires de la Révolution française, L'Histoire socialiste de Jaurès n'en a pas moins ouvert la voie à l'historiographie scientifique contemporaine de la révolution, elle est loin d'avoir perdu son élan créateur. C'est un triple héritage de science, de civisme et de foi que, relayé par Georges Lefebvre, Jaurès nous a transmis. C'est ce triple héritage que nous entendons maintenir et promouvoir $»^{65}$.

Pour Soboul, l'héritage en question ne rencontre pas seulement ses propres aspirations politiques et ses convictions d'historien, mais représente en grande partie le socle sur lequel il s'est appuyé pour s'élever dans la carrière universitaire. Notons toutefois que malgré son œcuménisme apparent, l'héritage historiographique défendu par Soboul laisse apparaître une distance vis-à-vis de Mathiez. Question de génération et question de conception du travail historique : si Soboul a consacré dix ans de sa vie à l'écriture d'une thèse d'histoire politique, il est avant tout un élève de Lefebvre. Historiographiquement parlant, son point de vue «par en-bas» s'oppose fondamentalement à la démarche mathiezienne. Maisplus encore, c'est au regard du déroulement de leurs carrières respectives que se creuse la distance entre Soboul et Mathiez, qu'il faut lire à l'aune des parcours différenciés de Lefebvre et de Mathiez ${ }^{66}$.

L'attachement soboulien à une pratique très commémorative de l'historiographie laisse peu de place à d'autres lectures et à d'autres approches. L'ouverture de la revue s'observe surtout au cours de la deuxième période de la gestion Soboul et se manifeste essentiellement par l'apparition progressive d'articles consacrés à l'étude du discours politique issus des recherches menées par le laboratoire de lexicologie politique de l'École normale supérieure de Saint-Cloud. L'arrivée des travaux vovelliens et autres réflexions se plaçant sur le terrain de l'histoire culturelle est plus discrète encore.

Homme de défi, Albert Soboul se lance néanmoins dans d'autres types d'ouvertures. En 1975, il cède ainsi un numéro entier des AHRF aux responsables de la petite Société d'histoire de 1848 que dirigeait depuis peu Maurice Agulhon et dont les moyens étaient trop limités pour envisager la création d'une revue. Avançant l'argument des liens d'une Révolution à l'autre et de l'intérêt qu'avait toujours porté Georges Lefebvre à l'histoire des révolutions du XIX ${ }^{e}$ siècle, Soboul envisagea même de répéter l'expérience régulièrement. À l'occasion du colloque organisé pour le centenaire de la Société de 1848 en 2005, Maurice Agulhon a rappelé combien l'initiative fut décisive, redonnant à l'histoire de 48 toute sa place dans le concert des révolutions du XVIII ${ }^{\mathrm{e}}$ et XIX ${ }^{\mathrm{e}}$ siècle. Elle devait malheureusement prendre fin de façon abrupte quelques mois plus tard.

\section{Fatigue, lassitude et désillusions : l'impossible rupture épistémologique}

41 De nombreux témoignages concordent pour dire qu'au cours des années 1970, Albert Soboul s'est attaché de plus en plus à une épistémologie historiciste, scientiste, développant même une sorte de réflexe anti-intellectualiste, comme si l'abstraction l'agaçait, comme si elle éloignait forcément les chercheurs de la recherche érudite fondée sur les sources à laquelle il tenait tant. Son séminaire avait pourtant largement 
bénéficié du regain d'intérêt pour la théorie dans la foulée de mai 1968. Il en sortait des travaux novateurs, qu'objectivement Soboul favorisait doublement: en leur donnant une tribune dans son séminaire et une visibilité dans sa revue. Néanmoins il n'y adhéra pas ou peu et en tint à peine compte dans ses dernières grandes synthèses. Dès lors, une question se pose : quelle fut la part de la fatigue, de la lassitude, celle des désillusions intellectuelles et politiques, celle enfin des certitudes fondées sur sa propre expérience de chercheur, dans son refus manifeste d'opérer pour lui-même, mais aussi pour la revue qu'il dirigeait, le virage épistémologique que beaucoup de ses disciples appelaient de leurs vœux?

S'il est difficile d'expliquer en quelques mots l'attitude de Soboul, au moins peut-on mentionner quelques éléments qui ne favorisèrent pas son aggiornamento. D'une part, Soboul était débordé. Outre ses cours, ses séminaires, ses étudiants, la direction des $A H R F$, il participait aux travaux de la Commission Jaurès, de la Commission internationale d'histoire de la Révolution, était sollicité à l'étranger, et ne manqua aucune des séances du Tribunal Russell $\mathrm{II}^{67}$. Ses conditions de travail n'étaient pas fameuses. De l'étranger, on pouvait imaginer l'institut comme une structure d'État confortablement dotée et la revue largement financée. Or Soboul n'a jamais cessé de rappeler à ses interlocuteurs dans quelle misère se trouvaient l'IHRF, la SER ou les AHRF. Ainsi confia-t-il un jour travailler " dans des conditions sous-hypo-artisanales " ${ }^{68}$. À la fatigue due aux tâches multiples qui lui incombaient s'ajoutait aussi, dans un contexte de déplacement irrépressible des intérêts des historiens vers des terrains qu'il ne maîtrisait pas, le poids des polémiques et des attaques ad hominem qu'il vivait d'autant plus mal que son caractère le portait à prendre toute chose très personnellement et il ne fut guère ménagé durant la fin de la décennie 1970. Nul besoin d'être Albert Soboul pour céder à la désolation face à l'une des agressions les plus surprenantes et les plus violentes dont il fit l'objet à l'occasion de la deuxième collaboration de la SER avec les historiens de 1848. Dans les dernières pages du numéro des AHRF d'avril-juin 1977 entièrement cédé à la Société d'histoire de 1848, l'équipe de rédaction avait cru bon de reproduire le compte rendu par André Zysberg du recueil d'Albert Soboul, Problèmes paysans, tout juste publié69. Bien connu des spécialistes de l'histoire maritime, Zysberg ne s'était jusqu'alors jamais fait remarquer pour sa connaissance de la question paysanne. Soboul, dont la thèse complémentaire avait porté sur les campagnes montpelliéraines à la fin de l'Ancien Régime ${ }^{70}$ et qui n'avait jamais cessé de s'intéresser à l'histoire rurale de la France, put lire dans sa propre revue qu'on s'étonnait de «l'indigence de sa pensée économique », "qu'[il] [écrivait] toujours laborieusement le même livre [...] depuis une vingtaine d'années » et que son recueil n'était rien d'autre que la « caverne d'Ali Baba » ${ }^{71}$. Inutile de dire que ce compte rendu grotesque, inopportun, mais non moins offensant, mit un terme définitif à la collaboration jusque-là amicale mise en place par Soboul entre les AHRF et la Société de $1848^{72}$.

43 Quand, au cœur de l'été 1982, Albert Soboul, qui a déjà subi deux accidents cardiaques, fait part à son ami Suratteau d'une grande lassitude intellectuelle et d'un état de fatigue généralisée, on mesure combien les dernières années de sa vie ont été difficiles $^{73}$. L'épilogue ne serait pas aussi tragique si à la maladie ne s'était ajouté l'abattement moral, résultat de bien des affrontements polémiques qui tout au long des années 1970 ont porté sur son nom. S'ils ont porté sur son nom, c'est à la fois que Soboul a souvent endossé la responsabilité de la défense de "l'historiographie 
classique » de la Révolution française face aux historiens qui avaient décidé de rompre avec les travaux de leurs devanciers; mais aussi, nous l'avons montré, que de nombreux discours l'ont enfermé dans la figure du gardien de la tradition ${ }^{74}$.

Bien que personnellement très exposé dans les débats, il préserva toujours la SER et les $A H R F$, refusant catégoriquement de les instrumentaliser à son propre profit. Dès lors, s'étonnera-t-on que Soboul ait été rarement désavoué dans ses choix par les membres de la SER ? Il semble que les controverses n'ont pas eu d'emprise sur son rapport aux instances robespierristes. Fidèle à l'œuvre de ses prédécesseurs, il ne transigea jamais et ne cessa d'insister, pendant près de quarante années, sur la spécificité originelle qui faisait de la création de Mathiez certes une association savante au recrutement largement académique, mais aussi un lieu de sociabilité rassemblant des hommes et des femmes venus d'horizons divers autour du combat toujours réactualisé que constitue la connaissance de Robespierre.

\section{NOTES}

1. Maurice Agulhon, « Ehistorien des sans-culottes », Le Monde, mardi 14 septembre 1982.

2. Jean-René Suratteau, Annales Historiques de la Révolution Française (AHRF), 1982, p. 323.

3. À sa mort, outre la co-présidence de la Société des études robespierristes dont il était également le secrétaire général en charge de la gestion des Annales historiques de la Révolution française, Albert Soboul dirigeait l'Institut d'histoire de la Révolution française, assurait le secrétariat général de la Commission internationale d'histoire de la Révolution française près le Comité international des sciences historiques et le secrétariat de la Commission pour la recherche et la publication des documents relatifs à la vie économique de la Révolution française dite "Commission Jaurès ». Il participait également au Conseil d'administration de la Société d'histoire moderne dont il assura la vice-présidence en 1966 et la présidence en 1968.

4. AlfredCobban, The Myth ofthe French Revolution, Londres, University College, 1955, 25 p.

5. Jacques Godechot et Robert Palmer, "Le problème de l'Atlantique du XVIIIe au XXe siècle ", Relazioni del X Congresso Internazionale di Scienze Storiche (Rome 4-11 septembre 1955), vol.5, Florence, 1955, p. 175-239.

6. François Furet et Denis Richet, La Révolution française, vol. 1, Des états généraux au 9 thermidor, Paris, Hachette, 1965, 372 p.

7. François Furet, « Le catéchisme révolutionnaire », Annales ESC, 1971, p. 255-289.

8. Pour rédiger cet article nous nous sommes appuyés principalement sur la correspondance d'Albert Soboul avec Jean-René Suratteau, conservée jusqu'à ce jour par la famille de Jean-René Suratteau. Qu'il nous soit permis de remercier ici Jean-François Suratteau pour son aide précieuse et désintéressée.

9. L'histoire de la SER n'a jusqu'à présent fait l'objet que d'investigations limitées. Voir l'article de Maurice Dommanget, "La Société et les Annales. Cinquante ans d'histoire (1908-1958)", $A H R R F, 1958$, p. 6-27, publié en 1958 à l'occasion du cinquantenaire de la SER et consacré en grande partie aux années d'avant-guerre. Plus récemment, dans le cadre d'un mémoire de master soutenu en 2006 à l'université de Rouen, Timothée Roquigny s'est attelé à un immense travail de dépouillement des AHRRF et a proposé une analyse statistique poussée de leur contenu: 
Timothée Roquigny, La Société des études robespierristes et les Annales historiques de la Révolution française de 1958 à 2005 (dir. Michel Biard).

10. En un siècle d'existence, les activités régulières de la SER et la publication trimestrielle des AHRRF n'ont été suspendues qu'au cours de la guerre et de l'occupation, soit entre juin 1940 et le 21 janvier 1945 pour les Assemblées générales de la Société et entre juin 1940 et février 1946 pour la parution de la revue (voir dans le présent numéro l'article consacré à cette période par Claude Mazauric, « La SER dans la tourmente : 1940-1945 »).

11. Sinon la correspondance privée, source précieuse mais par définition dispersée et dont nous avons pu constater qu'elle est encore en grande partie inaccessible à l'historien, voire tout simplement perdue.

12. Le colloque tenu en 1992 à Clermont-Ferrand auquel participèrent notamment Claude Mazauric, Michel Vovelle, Matthias Middell et Pascal Dupuy et dont les actes ont été publiés en 1994 dans le Bulletin d'histoire de la Révolution française du CTHS constitue un jalon important dans la constitution d'une réflexion historienne sur l'apport de Soboul à l'historiographie révolutionnaire. Cependant, situé explicitement dans une logique commémorative - dix ans après la mort de l'historien - ce colloque n'avait pas pour objet de revenir sur la trajectoire de Soboul de façon critique et renouvelée. La remarque vaut également pour la journée organisée à Nîmes en 2002 - encore un anniversaire -, publiée sous le titre Colloque Albert Soboul, vingt ans après. Des gens de métiers aux Sans-culottes,XviIIe-début $\mathrm{xIx}^{\mathrm{e}}$ siècle, Nîmes, Société d'histoire moderne et contemporaine de Nîmes et du Gard, 2004, 159 p. Le livre de Claude Mazauric, Albert Soboul, Un historien en son temps, essai de biographie intellectuelle et morale, Aubenas, Éditions d'Albret, 2003, $253 \mathrm{p}$. est à ce jour la tentative la plus aboutie de cerner le personnage d'un point de vue biographique tout en s'attachant à expliciter les ancrages intellectuels de son œuvre. Nous reviendrons sur les problèmes propres à ce texte dans notre développement. Pour un survol rapide de la vie de Soboul agrémenté de détails bibliographiques on pourra se reporter par exemple à l'article de James Friguglietti, «the French Revolution Seen From the Left: Albert Soboul as a Historian ", dans John F.Sweers (ed), Proceedings of the Annual Meeting of the Western Society for French History, Albuquerque, 24-27 october 1984, rhe university of Kansas, Lawrence, Kansas, 1985, p. 100-107.

13. Le dépouillement des AHRF réalisé par Timothée Roquigny, op. cit., permet de quantifier à la ligne près les textes signés par Soboul dans la revue tout en identifiant précisément aussi bien le type de ses interventions (article, compte rendu...) que la problématique dominante (histoire politique, histoire sociale, etc.). De même, la bibliographie établie par Françoise Brunel après la mort de Soboul et publiée à l'occasion de la réédition du Précis d'histoire de la Révolution française aux Éditions sociales, présente de l'œuvre de Soboul un relevé des plus exhaustifs auquel ne manquent que les articles, comptes rendus et éditoriaux publiés dans la presse généraliste.

14. Nous ne pouvons aborder ici qu'un choix nécessairement restreint de discours et limiter l'analyse aux catégories principales. Nous développerons notre étude des discours dans notre thèse de doctorat actuellement en cours sur l'historiographie jacobine de la Révolution française.

15. François Furet, op. cit.

16. François Furet et Denis Richet, op. cit. Au sujet de la réception de ce livre par les historiens de la Révolution française, voir notre article : Julien Louvrier, « Penser la controverse. La réception du livre de François Furet et Denis Richet, La Révolution française », AHRF, 2008-1, p. 151-176. Rappelons que le livre en question fit l'objet d'une critique en deux temps. Se plaçant sur le plan universitaire, Claude Mazauric publia d'abord dans les AHRF en 1967 une longue note critique se voulant avant tout académique et érudite. Trois ans plus tard, usant d'un ton militant et outrancier, Mazauric révisa son texte, dans un sens cette fois délibérément politique et l'inséra dans le recueil sur la Révolution française (Paris, Éditions Sociales, 1970, 238 p.) que préfaça Soboul. Il ne s'agissait plus, comme en 1967, de présenter aux historiens spécialistes de la Révolution les incohérences et les erreurs que comportait le récit de Furet et Richet, mais bien de dénoncer à un 
public plus large, lecteur des ouvrages des Éditions sociales, les visées politiques de l'interprétation de la Révolution proposée par les auteurs. Chronologiquement, le « discours de polémique " développé par Furet contre Soboul constitue une réponse au texte de Claude Mazauric dans sa version augmentée de 1970.

17. François Furet, « Le catéchisme révolutionnaire », article cité, p. 261.

18. ibid.

19. ibid.

20. ibid, p. 262.

21. Ibid, p. 263

22. ibid, p. 271-272.

23. François Furet, Penser la Révolution française, Paris, Gallimard, 1978, 259 p.

24. Le nouvel observateur, $\mathrm{n}^{\circ} 932,18-25$ septembre 1982, p. 55.

25. De ce point de vue, l'article nécrologique publié dans Le Monde est beaucoup plus nuancé. À ce sujet, il est utile de consulter le dernier chapitre du recueil publié par Maurice Agulhon, Histoire et politique à gauche, Paris, Perrin, 2005, 162 p., p. 126-142. L'auteur revient précisément sur les circonstances qui l'ont amené à rédiger la notice nécrologique en question.

26. Michael Scott Christofferson, French Intellectuals against the Left: the Antitotalitarian Moment of the 1970s, New York, Berghahn Books, 2004, X-294 p.

27. Pour l'analyse du phénomène de «disqualification symbolique du communisme» dont l'histoire reste à faire, voir l'article programmatique de Bernard Pudal, «La beauté de la mort communiste ", Revue française de science politique, vol. 52, n 5-6, oct-déc 2002, p. 545-559.

28. Une analyse sociologique fine des acteurs des controverses historiographiques sur la Révolution française révèlerait que se joue là aussi, au-delà d'une lutte politique et derrière les arguments scientifiquement élaborés, une vraie lutte de classes.

29. François Furet, «Histoire universitaire de la Révolution française », dans François Furet et Mona Ozouf (dir.), Dictionnaire critique de la Révolution française, Paris, Flammarion, 1988, 1122 p., p. 979-987.

30. Ibid, p. 997 : «Resté longtemps solitaire, extérieur à cette voie [l'enfermement dans un système d'interprétation rigide et pauvre du marxisme-léninisme greffé sur le positivisme républicain], Georges Lefebvre a élaboré dans les premières décennies du siècle ce qui reste de plus original de son œuvre : une histoire rurale de la Révolution. Mais quand la mort de Mathiez lui ouvre la voie de la magistrature suprême [la chaire d'histoire de la Révolution française à la Sorbonne] lui aussi se soumet à ce lit de Procuste méthodologique conçu par son prédécesseur ; et la conjoncture politique de la Libération fera le reste [...]. C'est la dernière étape de cette tradition universitaire de l'historiographie révolutionnaire " ( $\mathrm{NdA}$, c'est nous qui soulignons).

31. Ceci étant dit, les arguments qu'a pu avancer Furet dans l'approfondissement de son réquisitoire contre Soboul ne sont pas tous irrecevables ; le problème est qu'ils n'ont pas eu pour seule fonction de produire un savoir scientifique neuf mais de justifier des positions dans un débat polémique entre historiens qui débordait beaucoup le strict cadre de la discipline. Il est tout à fait concevable de parler objectivement, par exemple du marxisme de Soboul, mais cela nécessite de produire en amont un travail visant à replacer l'importance de la scolastique marxiste dans les études historiques en France dans les années 1960 et 1970 et plus généralement de questionner la façon dont on concevait alors la science sociale. or ce travail préalable de construction de l'objet « histoire universitaire de la Révolution française » n'a pas été fait, du moins pas par les historiens de la Révolution française qui entendaient substituer une nouvelle interprétation du phénomène révolutionnaire à l'explication classique alors dominante.

32. Claude Mazauric, op. cit.

33. Le « discours de combat » et le "discours de commémoration » ne se réduisent aucunement à ces deux exemples. Pour des raisons matérielles nous sommes néanmoins contraints de limiter 
notre analyse à ces deux points qui nécessiteraient eux aussi de plus longs développements (cf. note 14).

34. Albert Soboul, Comprendre la Révolution, Paris, La Découverte, 1981, p. 325.

35. Il faudrait réfléchir aux raisons qui ont poussé Claude Mazauric à ne pas approfondir sa réflexion sur les positions de Soboul vis-à-vis du Parti communiste, notamment de ne pas éclairer les circonstances et les raisons de ses choix répétés, surtout dans les dernières années de sa vie, de s'opposer à la direction du parti. Pourquoi ne rien dire par exemple de son soutien et de sa signature à l'appel lancé par la cellule Jacques Duclos de l'Université d'Aix en Provence au printemps 1978, dit «texte des 300 ». La figure du Soboul oecuménique dépeint par Mazauric semble devoir se conformer au point de vue de la mémoire communiste officielle et conserver sa pleine cohérence avec l'image de l'historien diffusée dans la presse communiste après sa mort.

36. Claude Mazauric dresse notamment la liste des femmes historiennes de la Révolution qui ont travaillé dans l'entourage de Soboul, celle des chercheurs qui sont intervenus dans son séminaire, ou encore celle des historiens étrangers qu'il fréquentait.

37. Il s'agit du titre du deuxième chapitre du livre.

38. Ibid, p. 86.

39. AHRF, 1982, p. 323.

40. En réalité, le fonctionnement de la SER impliquait alors que le secrétaire général prenne en charge la direction des AHRF. Il n'y avait donc rien d'exceptionnel à ce que Soboul cumule à la fois les fonctions de «secrétaire général » et «directeur " de la revue. Quant à la référence à la fonction de "rédacteur en chef ", elle est purement rhétorique dans la mesure où elle n'existait pas encore et n'était nullement prévue par les statuts.

41. Albert Soboul, op. cit., p. 326.

42. Albert Soboul, 1789. L'An I de la liberté, Paris, Éditions Sociales internationales, 1939, 303 p.

43. AHRF, 1946, p. 86. Notons tout de même que Georges Lefebvre avait rendu compte dans les AHRF $\left(\mathrm{n}^{\circ} 16,1939\right)$ du Saint-Just, ses idées politiques et sociales, publié par Soboul aux Éditions Sociales internationales sous le pseudonyme de Pierre Derocles à l'occasion du centcinquantenaire de la Révolution. À cette époque Soboul et Lefebvre ne se connaissaient pas, mais Lefebvre pouvait-il ignorer que le jeune Soboul, auteur d'un DES sur Saint-Just dirigé par Philippe Sagnac, se cachait derrière le nom de Derocles?

44. Georges Lefebvre, "Une enquête collective ", AHRF, oct-déc 1947. Le volume sera publié en 1950 : Cuuvres de Maximilien Robespierre, Tome 6, Discours, Première partie, 1789-1790. Édition préparée sous la direction de Marc Bouloiseau, Georges Lefebvre, Albert Soboul, Paris, Presses universitaires de France, 1950, XXXII-704 p.

45. Il s'agit essentiellement de travail intellectuel : rédaction d'articles et de comptes rendus pour les publications du Parti communiste.

46. Albert Soboul, «Les "Institutions républicaines" de Saint-Just d'après les manuscrits de la Bibliothèque Nationale ", AHRF, 1948, n 3, p. 193-262.

47. Voir à ce sujet les explications que donne Soboul dans ses entretiens avec Raymond Huard et Marie-Josèphe Naudin publiés en annexe du livre de Claude Mazauric, op. cit, p. 161-232. Notons que dans les mois qui suivent la Libération, Soboul milite ardemment en faveur de « l'amalgame », c'est-à-dire de l'incorporation des FFI et FTP aux cadres de l'armée régulière. À ce titre, il faut comprendre le livre L'Armée nationale sous la Révolution, 1789-1794, Paris, Éditions France d'abord, 1945, 143 p., qu'il publie en 1945, moins comme l'œuvre d'un historien de la Révolution que comme une contribution politique chargée de fonder historiquement une revendication politique.

48. Albert Soboul, 1848, Paris, Union Nationale des Intellectuels, 1948.

49. Albert Soboul, La Révolution française, 1789-1799, Paris, Éditions sociales, 1948, 388 p.

50. Voir le compte rendu favorable par Georges Lefebvre des livres publiés par Soboul depuis la Libération dans une note bibliographique récapitulative, AHRF, 1950, p. 294. 
51. «Procès-verbal de l'assemblée générale du 12 juin 1949 », AHRF, juillet-septembre 1949, p. 286-287.

52. Albert Soboul, « Correspondance », AHRF, mai-juin 1958, p. 91-93. «L'histoire de la Révolution ne s'est pas arrêtée à Mathiez, ni la sociologie à Durkheim. Ce n'est pas manquer à leur mémoire, ni attacher "l'opprobre" à leurs noms, que de partir, en bonne méthode, de leurs travaux pour essayer d'aller plus avant dans la connaissance ».

53. Lettre de Robert Schnerb à Maurice Dommanget, 2 mars 1959, Archives nationales, IFHS, Fonds Maurice Dommanget, carton 14/AS/333, $\mathrm{n}^{\circ} 389$.

54. Lettre de René Garmy à Maurice Dommanget, 29 septembre 1959, Archives nationales, IFHS, Fonds Maurice Dommanget, carton 14/AS/378, $\mathrm{n}^{\circ} 23$.

55. Lettre d'Albert Soboul à Jean-René Suratteau, 8 septembre 1959, archives privées de la famille Suratteau.

56. Entre 1959 et 1968 Soboul ne publie pas moins de huit articles dans les AHRF.

57. Julien Louvrier, «Penser la controverse », article cité.

58. Soboul fut particulièrement irrité par l'affaire du " plagiat » dont Furet et Richet l'accusèrent. Pour les détails de cette affaire nous renvoyons à notre article « Penser la controverse ».

59. Lettre d'Albert Soboul à Jean-René Suratteau, 17 octobre 1959, archives privées de la famille Suratteau.

60. Diversification que permettent d'observer très précisément les tableaux réalisés par Timothée Roquigny, op. cit.

61. MarcelReinhard, «Compte rendu du livre de René Sidersky, Confidences révolutionnaires, Paris, Clavreuil, 1967, 38 p. », AHRF, 1970, p. 407.

62. Lettre d'Albert Soboul à Jean-René Suratteau, 29 octobre 1959, archives privées de la famille Suratteau.

63. Compte rendu de l'assemblée générale de la SER du 11 mars 1962, AHRF, 1962, p. 255-256. Une position qui n'est pas seulement de principe comme le confirme ce courrier envoyé par Soboul à l'érudit d'Abbeville Robert Legrand alors que celui-ci vient de favoriser l'adhésion d'une de ses connaissances: " Nous sommes très heureux d'accueillir M. Jean Delattre dans notre société, dîtes le lui. Il faut que les érudits régionaux nous rejoignent [...] », Lettre de Soboul à Robert Legrand, 12 septembre 1965, archives privées de la famille Legrand. Les positions de Soboul sont de ce point de vue très proches de celles que défend Maurice Dommanget sur le même sujet, au cours de l'année 1964 (AHRF, p. 253-254) et que défendait déjà Mathiez.

64. Lettre d'Albert Soboul à Jean-René Suratteau, archives privées de la famille Suratteau. Au cours de ses premières années en tant que secrétaire général de la SER, Soboul ne se sent pas particulièrement soutenu. S'adressant au responsable de la revue italienne Rivista storica del socialismo en janvier 1962, il se confie au détour d'une phrase : «moi aussi, je dirige une revue (où je suis obligé de tout faire moi-même) ", Lettre d'Albert Soboul à Luigi Cortesi, archives de la Rivista storica del socialiso conservées à la Fondation Lelio Basso, Rome.

65. Albert SoBоUL, « Hommage à Georges Lefebvre », AHRF, juillet-septembre 1979, p. 454.

66. Le rapport de Soboul à la mémoire de l'historiographie révolutionnaire est une problématique en soi que nous ne pouvons développer ici.

67. Sur l'activité de Soboul au sein du Tribunal Russell II, quelques éléments dans notre article, Julien LOUVRIER, « Le Tribunal Russell II pour l'Amérique Latine : mobiliser les intellectuels pour sensibiliser l'opinion publique internationale », dans Emmanuel SolERs (dir.), Les intellectuels dans la cité. Actes du colloque international de Rouen, 19-21 mai 2005, à paraître aux PURH.

68. Lettre d'Albert Soboul à Lelio Basso, 14 février 1974, archives de la Fondation Lelio Basso, Rome.

69. Albert SовоUL, Problèmes paysans de la Révolution, 1789-1848, Paris, Maspéro, 1976, 442 p.

70. Albert SоBOUL, Les campagnes montpelliéraines à la fin de l'Ancien Régime: propriété et cultures d'après les compoix, Paris, PUF, 1958, 157 p. 
71. AHRF, 1977, avril-juin, p. 340.

72. Dans sa communication au colloque du centenaire de la Société de 1848, Maurice Agulhon revient sur cette affaire mais ne révèle pas toutes les étapes qui conduirent à ce sabordage et dont on ne peut croire que la direction de la Société n'ait été complice. Voir Maurice AGULHoN, «Le rétablissement. Huit années d'expériences, 1974-1982", Revue d'histoire du XIXe siècle, 2005-31, La «Société de 48 » a cent ans, [En ligne], mis en ligne le 18 février 2006. URL : http :// rh19.revues.org/ document949.html. Consulté le 22 janvier 2008 : «Les actes du colloque sur Foucault ont été publiés dans les AHRF, par notre équipe, donc, de chercheurs quarante-huitards. Or, dans ce nombre figurait Zysberg qui publiait un fragment de texte très hostile et méchant sur Albert Soboul. Pauvre Albert Soboul, oserai-je dire ! Il y avait certainement dans son parti (le PCF) des gens qui le trouvaient trop complaisant pour moi, qui après tout, était un « renégat » du PC, un peu libéral donc, autant dire un peu droitier. Mais d'autres, du bord opposé, le percevaient encore comme un pur stalinien et l'insultaient comme tel! Sur cette affaire, nous avons divorcé (à l'amiable - cela arrive...). Ce conflit de polémique politique cumulait aussi ses effets avec des difficultés de cotisations, matérielles ; même aux AHRF tout le monde ne devait pas être d'accord pour céder 1 numéro sur 4 de la revue de la Grande Révolution aux minores de 48. ».

73. Dernière lettre d'Albert Soboul à Jean-René Suratteau, 26 juillet 1982. Lettre de 3 feuillets non transmise par la famille de Jean-René Suratteau mais recopiée partiellement par Jean-François Suratteau dans l'inventaire qu'il a réalisé de la correspondance de son père et que je cite ici : « [...] tu me donnais aussi des nouvelles de ta santé, précisant que depuis ton opération tu n'avais pas récupéré. J'espère que de bonnes et longues vacances te remettront sur pied. Peut-être en astu trop fait, il faut savoir élaguer, sans toutefois lâcher la rampe. Je te parle d'expérience. Moi non plus je n'ai pas récupéré depuis mon second accident cardiaque (janvier 1981). Fatigue extrême, baisse de dynamisme, lassitude intellectuelle... J'ai abandonné tout ce qui n'était pas essentiel -comités, réunions, commissions... Je me couche tôt et me lève tard. Cela n'a pas empêché au début de juillet, que je sois éreinté, excédé... Me voici donc à Nîmes, où il fait une chaleur accablante... ».

74. Albert SовоUL, « Historiographie révolutionnaire classique et tentatives révisionnistes ", dans Albert SoBoul, Comprendre la Révolution, p. 323-345.

\section{RÉSUMÉS}

Retracer la trajectoire d'Albert Soboul impose de rompre avec les discours qui l'ont enfermé dans la figure figée de l'historien marxiste dogmatique ou dans celle du défenseur œcuménique d'une tradition historiographique. Une fois dégagées des lectures polémiques les réalités de la vie de Soboul se laissent appréhender plus aisément, révélant une dynamique singulière, mêlant engagements politique et historiographique, carrière académique et enjeux liés à la sociabilité robespierriste. Deux périodes rythment son action dans la SER. La première (1946-1958) est marquée par les certitudes et les accomplissements : rédaction de la thèse, premières activités au sein de la Société. La seconde (1958-1982) est synonyme de responsabilités, puis de désillusions. Secrétaire général de la SER, Soboul donne deux directions à son action : respect des orientations de Mathiez et défense de l'héritage de Lefebvre. Pris violemment à partie dans les débats des années 1970 qui sont des affrontements idéologiques, Soboul cherche à préserver les AHRF. Son 
caractère semble lui interdire tout aggiornamento. Au dynamisme des années 1960 succède une grande lassitude intellectuelle, indice de bien des désillusions.

To retrace the career of Albert Soboul requires discarding notions that imprisoned him into a cardboard figure of a dogmatic marxist historian or universal defender of an historiographical tradition. Once freed from such polemical interpretations, the realities of Soboul's life are more easily understood, revealing a singular energy, mixing political involvements, historiography, an academic career, and issues linked to a robespierrist sociability. Two periods mark his action in the SER. The first (1946-1958) is characterized by certainties and accomplishments: the writing of his thesis, the first activities of the organization. The second (1958-1982) is synonymous with responsibilities, then disillusionment. General Secretary of the SER, Soboul gave two directions to his action: respect for the position of Mathiez and defence of the heritage of Lefe-bvre. Violently involved in the debats of the 1970's, themselves essentially confrontations of ideology, Soboul endeavored to preserve the AHRF. His personality seemed to preclude any compromise. After the dynamism of the 1960's came a period of major intellectual lassitude, a sign of much disillusionment.

INDEX

Mots-clés : Albert Soboul, Société des études robespierristes, historiographie, discours, polémique, commémoration

\section{AUTEUR}

\section{JULIEN LOUVRIER}

GRHIS - Université de Rouen Rue Lavoisier 76821 Mont-Saint-Aignan julien.louvrier@gmail.com 